\title{
Telescopic Relative Entropy
}

\author{
Koenraad M.R. Audenaert \\ Department of Mathematics, \\ Royal Holloway, University of London, \\ Egham TW20 0EX, United Kingdom
}

\begin{abstract}
We introduce the telescopic relative entropy (TRE), which is a new regularisation of the relative entropy related to smoothing, to overcome the problem that the relative entropy between pure states is either zero or infinity and therefore useless as a distance measure in this case. We study basic properties of this quantity, and find interesting relationships between the TRE and the trace norm distance. We then exploit the same techniques to obtain a new and shorter proof of a lower bound on the relative Renyi entropies in terms of the trace norm distance, $\operatorname{Tr} \rho^{1-p} \sigma^{p} \geq 1-\|\rho-\sigma\|_{1} / 2$.
\end{abstract}

\section{Introduction}

The quantum relative entropy between two quantum states $\rho$ and $\sigma$, $S(\rho \| \sigma)=\operatorname{Tr} \rho(\log \rho-\log \sigma)$, is a non-commutative generalisation of the Kullback-Leibler distance between probability distributions. Because of its strong mathematical connections with von Neumann entropy, and its interpretation as an optimal asymptotic error rate in quantum hypothesis testing (in the context of Stein's lemma) relative entropy is widely used as a (non-symmetric) distance measure between states [7].

One of its drawbacks, however, is that for non-faithful (rank-deficient) states the relative entropy can be infinite. More precisely, the relative entropy is infinite when there exists a pure state $\psi$ such that $\langle\psi|\sigma| \psi\rangle$ is zero while $\langle\psi|\rho| \psi\rangle$ is not. In particular, relative entropy is useless as a distance measure between pure states, since it is infinite for pure $\rho$ and $\sigma$, unless $\rho$ and $\sigma$ are exactly equal (in which case it always gives 0 ).

There are various possibilities to overcome this deficiency. In [5], Lendi, Farhadmotamed and van Wonderen proposed a regularised relative entropy as

$$
R(\rho \| \sigma)=c_{d} S\left(\frac{\rho+\mathbb{1}_{d}}{1+d} \| \frac{\sigma+\mathbb{1}_{d}}{1+d}\right),
$$

where $d$ is the dimension, and $c_{d}$ is a normalisation constant. This only works for finite-dimensional states. 
Another possibility, also useful for infinite dimensional states, is to apply a smoothing process. One can define the smooth relative entropy between states $\rho$ and $\tau$ as the infimum of the ordinary relative entropy between $\rho$ and another state $\tau$, where $\tau$ is constrained to be $\epsilon$-close to $\sigma$ in trace norm distance:

$$
S_{\epsilon}(\rho \| \sigma)=\inf _{\tau}\left\{S(\rho \| \tau): \tau \geq 0, \operatorname{Tr} \tau \leq 1,\|\tau-\sigma\|_{1} \leq \epsilon\right\} .
$$

This form of smoothing has already been applied to Renyi entropies and max-relative entropy [39], giving rise to a quantity with an operational interpretation, but it could equally well be applied to ordinary relative entropy.

In the case of the ordinary relative entropy there is a simple canonical choice for $\sigma$ that achieves the same purpose of regularisation but without having to find the exact minimiser. Namely, we can take that $\tau$ that is collinear with $\rho$ and $\sigma$; i.e. $\tau=a \rho+(1-a) \sigma$ (with $\left.a=\epsilon /\|\rho-\sigma\|_{1}\right)$.

By operator monotonicity of the logarithm, we have

$$
\log (\tau)=\log (a \rho+(1-a) \sigma) \geq \log (a \rho),
$$

and, therefore,

$$
\begin{aligned}
S(\rho \| \tau) & =\operatorname{Tr} \rho(\log \rho-\log \tau) \\
& \leq \operatorname{Tr} \rho(\log \rho-\log (a \rho)) \\
& =-\log a .
\end{aligned}
$$

Thus, $S(\rho \| \tau)$ is bounded above by $-\log a$, which is finite for $0<a<1$. It therefore makes perfect sense to normalise $S(\rho \| \tau)$ by dividing it by $-\log a$, producing a quantity that is always between 0 and 1 .

These observations led us to define what we call the telescopic relative entropy (TRE), a particular regularisation of the ordinary relative entropy that is also defined in Hilbert spaces of infinite dimension:

Definition 1. For fixed $a \in(0,1)$, the a-telescopic relative entropy between states $\rho$ and $\sigma$ is given by

$$
S_{a}(\rho \| \sigma):=\frac{1}{-\log (a)} S(\rho \| a \rho+(1-a) \sigma) .
$$

Furthermore, we define

$$
\begin{aligned}
& S_{0}(\rho \| \sigma):=\lim _{a \rightarrow 0} S_{a}(\rho \| \sigma) \\
& S_{1}(\rho \| \sigma):=\lim _{a \rightarrow 1} S_{a}(\rho \| \sigma) .
\end{aligned}
$$

We'll show below that these limits exist. 
The origin of the name is that the operation $\sigma \mapsto a \rho+(1-a) \sigma$ acts like a 'telescope' with 'magnification factor' $1 /(1-a)$, bringing the state $\sigma$ closer to the 'vantage point' $\rho$ and bringing observed pairs of states $\sigma_{i}$ closer to each other.

The purpose of this paper is to initiate the study of this quantity. The telescoping operation $\sigma \mapsto a \rho+(1-a) \sigma$ and subsequent scaling of the relative entropy by $1 /(-\log a)$ may seem like a fairly innocuous operation, but has a number of far-reaching and sometimes unexpected consequences. Because of the linearity of the telescoping operation, the TRE inherits most of the desirable properties of the ordinary relative entropy. However, a host of additional relations in the form of sharp inequalities may be derived that in the case of the ordinary relative entropy simply make no sense, because the constants appearing in the inequality would be infinite. At the end of this paper, we briefly consider the telescoping operation in the context of the relative Renyi entropies. We exploit the same techniques used for the TRE to obtain a new and shorter proof of a lower bound on the relative Renyi entropies in terms of the trace norm distance, $\operatorname{Tr} \rho^{1-p} \sigma^{p} \geq 1-\|\rho-\sigma\|_{1} / 2[1]$.

\section{Preliminaries}

For any self-adjoint operator $X$ on a Hilbert space $\mathcal{H}$, we denote by $\operatorname{supp} X$ the support of $X$, i.e. the subspace of $\mathcal{H}$ which is the orthogonal complement of $\operatorname{ker} X$, the kernel of $X$. The projector on the support of $X$ will be denoted by $\{X\}$. We denote by $P_{X}$ the orthogonal projector from $\mathcal{H}$ onto $\operatorname{supp} X$, so that $P_{X}^{*}$ is the injection of supp $X$ back into $\mathcal{H}$. Thus $P_{X}^{*} P_{X}=\{X\}$. The compression of $A$ to the support of $X$, which we'll denote by $\left.A\right|_{X}$, is the operator with domain $\operatorname{supp} X$ given by

$$
\left.A\right|_{X}=P_{X} A P_{X}^{*}
$$

By definition, for any positive operator $X \geq 0$, we have $\left.X\right|_{X}>0$, strictly.

Two quantum states are mutually orthogonal, denoted $\rho \perp \sigma$, iff $\operatorname{Tr} \rho \sigma=0$.

For any self-adjoint operator $X, X_{+}$will denote the positive part $X_{+}=(X+|X|) / 2$. It features in an expression for the trace norm distance between states:

$$
T(\rho, \sigma):=\frac{1}{2}\|\rho-\sigma\|_{1}=\operatorname{Tr}(\rho-\sigma)_{+} .
$$

The trace of the positive part has the variational characterisation $\operatorname{Tr} X_{+}=$ $\max _{P} \operatorname{Tr} X P$, where the maximisation is over all self-adjoint projectors. Hence, for all such projectors $P, \operatorname{Tr} X P \leq \operatorname{Tr} X_{+}$. 
The Pinsker bound is a lower bound on the ordinary relative entropy in terms of trace norm distance, [7].

$$
S(\rho \| \sigma) \geq 2 T(\rho, \sigma)^{2} .
$$

No upper bound in terms of the trace norm distance is possible, because the relative entropy can be infinite.

We will also need the following integral representation of the logarithm: for $x>0$, we have

$$
\log x=\int_{0}^{\infty} d s\left(\frac{1}{1+s}-\frac{1}{x+s}\right) .
$$

This immediately provides an integral representation for the telescopic relative entropy:

$$
\begin{aligned}
& S_{a}(\rho \| \sigma) \\
& =\frac{1}{\log a} \int_{0}^{\infty} d s \operatorname{Tr} \rho\left[(\rho+s)^{-1}-(a \rho+(1-a) \sigma+s)^{-1}\right] \\
& =\frac{1}{\log a} \int_{0}^{\infty} d s \operatorname{Tr} \rho(\rho+s)^{-1}(1-a)(\sigma-\rho)(a \rho+(1-a) \sigma+s)^{-1} .(8)
\end{aligned}
$$

Another integral we will encounter is $\int_{0}^{\infty} d s x /(x+s)^{2}$. For $x=0$, the integral obviously gives 0 . For $x>0$ it gives 1 . Hence

$$
\int_{0}^{\infty} d s(\rho+s)^{-1} \rho(\rho+s)^{-1}=\{\rho\} .
$$

From integral representation (6) we get an expression for the Fréchet derivative of the matrix logarithm:

$$
\left.\frac{d}{d t}\right|_{t=0} \log (A+t \Delta)=\int_{0}^{\infty} d s(A+s)^{-1} \Delta(A+s)^{-1} .
$$

It will be useful to introduce the following linear map, for $A \geq 0$ :

$$
\mathcal{T}_{A}(\Delta)=\int_{0}^{\infty} d s(A+s)^{-1} \Delta(A+s)^{-1} .
$$

Thus

$$
\left.\frac{d}{d t}\right|_{t=0} \log (A+t \Delta)=\mathcal{T}_{A}(\Delta) .
$$

It's easy to check that for $A \geq 0, \mathcal{T}_{A}(A)=\{A\}$. Thus, for $A>0$, we have $\mathcal{T}_{A}(A)=\mathbb{1}$. 
From this integral representation it also follows that, for any selfadjoint $A, \mathcal{T}_{A}$ preserves the positive semidefinite order: if $X \leq Y$, then $\mathcal{T}_{A}(X) \leq \mathcal{T}_{A}(Y)$. By cyclicity of the trace, we see that the map $\mathcal{T}_{A}$ is selfadjoint: $\operatorname{Tr} B \mathcal{T}_{A}(\Delta)=\operatorname{Tr} \Delta \mathcal{T}_{A}(B)$. Moreover, the map is positive semidefinite, in the sense that $\operatorname{Tr} \Delta \mathcal{T}_{A}(\Delta)$ is positive for any self-adjoint $\Delta$. This follows from the integral representation and the fact that for positive $X$ and self-adjoint $Y, \operatorname{Tr} X Y X Y=\operatorname{Tr}\left(X^{1 / 2} Y X^{1 / 2}\right)^{2} \geq 0$.

\section{Basic properties of Telescopic Relative Entropy}

From the discussion in the Introduction, we recall that the value of the telescopic relative entropy is always between 0 and 1, even for non-faithful states. Furthermore, it inherits many desirable properties from the ordinary relative entropy: positivity, the fact that it is only zero when $\rho$ and $\tau$ are equal (provided $a>0$ ), joint convexity in its arguments, and monotonicity under CPT maps.

As we do not restrict the arguments of the telescopic relative entropy to states, the definition is also applicable (in a useful way) to non-negative scalars:

$$
S_{a}(b \| c)=\frac{b(\log b-\log (a b+(1-a) c))}{-\log a} .
$$

For illustrative purposes, we graph the telescopic relative entropy for a variety of qubit state pairs, in figures 1 and 2 .
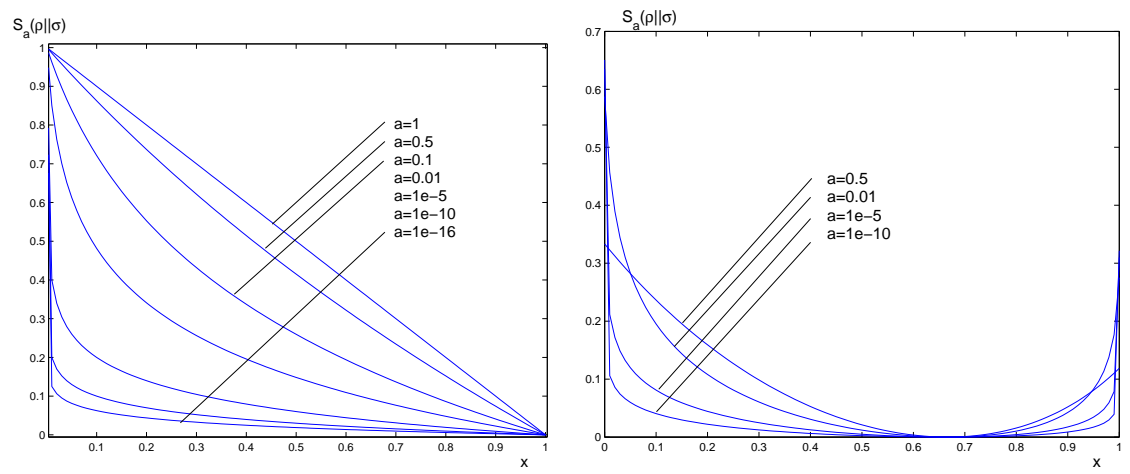

Fig. 1. (a) Telescopic relative entropy $S_{a}(\rho \| \sigma)$ between state $\rho=|0\rangle\langle 0|$ and state $\sigma=x|0\rangle\langle 0|+(1-x)| 1\rangle\langle 1|$, with $x$ ranging from 0 to 1 , and for various values of $a$; (b) same but for $\rho=(2 / 3)|0\rangle\langle 0|+(1 / 3)| 1\rangle\langle 1|$. 

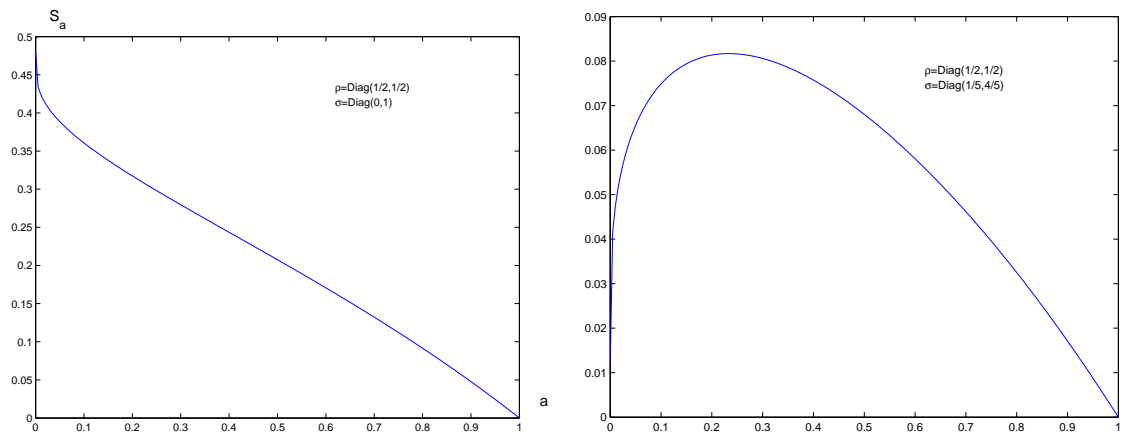

Fig. 2. (a) Telescopic relative entropy $S_{a}(\rho \| \sigma)$ between state $\rho=\mathbb{1}_{2} / 2$ and state $\sigma=|1\rangle\langle 1|$, with $a$ ranging from 0 to 1 ; (b) same but for $\sigma=$ $(|0\rangle\langle 0|+4| 1\rangle\langle 1|) / 5$.

\section{$3.1 \quad S_{0}$ and $S_{1}$}

One might think that the 1-telescopic relative entropy would be quite useless, because for $a=1, S(\rho \| a \rho+(1-a) \sigma)=S(\rho \| \rho)=0$. Nevertheless, it is a non-trivial quantity due to the normalisation by $1 /(-\log a)$. Likewise, one might mistakenly think $S_{0}$ is essentially the ordinary relative entropy; it is far from it, and for the same reason. Indeed, for any pair of states with finite ordinary relative entropy, e.g. when both states are faithful, $S_{0}$ is 0 , due to the normalisation. The 0 -telescopic relative entropy shows its true colours exactly in those cases when the ordinary relative entropy yields $+\infty$.

In fact, for $S_{0}$ and $S_{1}$ we have the following closed form expressions:

Theorem 1. For any pair of states $\rho, \sigma$,

$$
\begin{aligned}
& S_{0}(\rho \| \sigma)=1-\operatorname{Tr} \rho\{\sigma\} \\
& S_{1}(\rho \| \sigma)=1-\operatorname{Tr} \sigma\{\rho\}
\end{aligned}
$$

In particular, when $\sigma$ is pure, $S_{0}(\rho \| \sigma)=1-\operatorname{Tr} \rho \sigma$, and when $\rho$ is pure, $S_{1}(\rho \| \sigma)=1-\operatorname{Tr} \rho \sigma$. When $\sigma$ is faithful, $S_{0}(\rho \| \sigma)=0$; when $\rho$ is faithful, $S_{1}(\rho \| \sigma)=0$.

Proof. Consider first the limit $a \rightarrow 1$. Using de l'Hôpital's rule we find

$$
\lim _{a \rightarrow 1} \frac{1-a}{-\log a}=1
$$


Hence, by representation (8),

$$
\lim _{a \rightarrow 1} S_{a}(\rho \| \sigma)=-\int_{0}^{\infty} d s \operatorname{Tr} \rho(\rho+s)^{-1}(\sigma-\rho)(\rho+s)^{-1} .
$$

Therefore, from (9) we get the required

$$
\lim _{a \rightarrow 1} S_{a}(\rho \| \sigma)=-\operatorname{Tr}(\sigma-\rho)\{\rho\}=1-\operatorname{Tr} \sigma\{\rho\} .
$$

For the limit $a \rightarrow 0$ some more work is needed. Let us w.l.o.g. assume that $(\rho+\sigma) / 2$ is faithful; otherwise we take the compression of $\rho$ and $\sigma$ to the support of $(\rho+\sigma) / 2$. Again we use an integral representation, but in its more basic form (7). To calculate the limit $a \rightarrow 0$ we apply de l'Hôpital's rule to the whole expression and get

$$
\begin{aligned}
& S_{0}(\rho \| \sigma) \\
& =\lim _{a \rightarrow 0} a \frac{d}{d a} \int_{0}^{\infty} d s \operatorname{Tr} \rho\left[(\rho+s)^{-1}-(a \rho+(1-a) \sigma+s)^{-1}\right] \\
& =\lim _{a \rightarrow 0} \int_{0}^{\infty} d s \operatorname{Tr} a \rho(a \rho+(1-a) \sigma+s)^{-1}(\rho-\sigma)(a \rho+(1-a) \sigma+s)^{-1} \\
& =\lim _{a \rightarrow 0} \int_{0}^{\infty} d s \operatorname{Tr}(\rho-\sigma)(a \rho+(1-a) \sigma+s)^{-1} a \rho(a \rho+(1-a) \sigma+s)^{-1} .
\end{aligned}
$$

Here, the first factor $a$ comes from the derivative of $\log a$.

Because of our assumption that $(\rho+\sigma) / 2$ is faithful, $a \rho+(1-a) \sigma$ is faithful for any $a \in(0,1)$. Therefore, the integral

$$
\int_{0}^{\infty} d s(a \rho+(1-a) \sigma+s)^{-1}(a \rho+(1-a) \sigma)(a \rho+(1-a) \sigma+s)^{-1}
$$

yields the identity operator $\mathbb{1}$. Using this fact, we can rewrite our last expression for $S_{0}$ as

$$
\begin{aligned}
& S_{0}(\rho \| \sigma) \\
& =\lim _{a \rightarrow 0} \operatorname{Tr}(\rho-\sigma)\left[\mathbb{1}-\int_{0}^{\infty} d s\right. \\
& \left.\quad \quad(a \rho+(1-a) \sigma+s)^{-1}(1-a) \sigma(a \rho+(1-a) \sigma+s)^{-1}\right] \\
& =\operatorname{Tr}(\rho-\sigma)\left[\mathbb{1}-\int_{0}^{\infty} d s(\sigma+s)^{-1} \sigma(\sigma+s)^{-1}\right] \\
& =\operatorname{Tr}(\rho-\sigma)(\mathbb{1}-\{\sigma\}) \\
& =1-\operatorname{Tr} \rho\{\sigma\},
\end{aligned}
$$

as required. 


\subsection{Pure states}

From Theorem 1 we can derive the equalities

$$
S_{0}(\rho \| \sigma)=S_{1}(\rho \| \sigma)=T(\rho, \sigma)^{2},
$$

for pure $\rho$ and $\sigma$.

In fact, when $\rho$ and $\sigma$ are pure, there is a one-to-one relation between $S_{a}(\rho \| \sigma)$ and $T(\rho, \sigma)$ for any value of $a \in[0,1]$. Although the relation is somewhat complicated, in practice it shows that $S_{a}(\rho \| \sigma)$ is only slightly bigger than $T(\rho, \sigma)^{2}$ for $a \in(0,1)$.

Theorem 2. Let $\rho, \sigma$ be two pure states with trace norm distance $t=$ $\|\rho-\sigma\|_{1} / 2$. Then, for $a \in(0,1)$,

$$
S_{a}(\rho \| \sigma)=\frac{1}{-2 \log a}\left(-\log \frac{w}{4}-\frac{1-w /(2 a)}{\sqrt{1-w}} \log \frac{1+\sqrt{1-w}}{1-\sqrt{1-w}}\right),
$$

where

$$
w:=4 a(1-a) t^{2}
$$

Proof. By a suitable unitary transformation, the problem can be transformed to a two-dimensional one, with in particular

$$
\rho=\left(\begin{array}{ll}
1 & 0 \\
0 & 0
\end{array}\right), \quad \sigma=\left(\begin{array}{cc}
1-t & \sqrt{t(1-t)} \\
\sqrt{t(1-t)} & t
\end{array}\right) .
$$

The telescopic relative entropy is then given by

$$
S_{a}(\rho \| \sigma)=\frac{1}{-\log a}(-\log (a \rho+(1-a) \sigma))_{1,1}
$$

and after some basic calculations this reduces to the given formula.

For example, let $\rho$ and $\sigma$ be two pure two-level states, with the angle between their respective Bloch vectors equal to $\theta$. Since their trace norm distance is equal to $t=|\sin (\theta / 2)|$, we have $w=2 a(1-a)(1-\cos \theta)$.

\section{Comparison to trace norm distance}

In this section, we provide bounds on the telescopic relative entropy in terms of the trace norm distance.

It's very easy to derive a lower bound from the Pinsker lower bound on the ordinary relative entropy (5). 
Theorem 3. For two quantum states $\rho, \sigma$,

$$
S_{a}(\rho \| \sigma) \geq \frac{(1-a)^{2}}{-\log (a)} 2 T(\rho, \sigma)^{2} .
$$

Proof. Noting that $T(\rho, \tau)=(1-a) T(\rho, \sigma)$, this is a trivial consequence of the bound $S(\rho \| \tau) \geq 2 T(\rho, \tau)^{2}$.

While there is no upper bound on the ordinary relative entropy in terms of the trace norm distance, we can find an upper bound on the telescopic relative entropy. This bound has a very simple form, but is nevertheless the strongest one possible.

Theorem 4. With $\tau=a \rho+(1-a) \sigma$,

$$
S(\rho \| \tau) \leq-\log (a) T(\rho, \sigma) .
$$

This theorem immediately gives our first important relation for the TRE.

Corollary 1. For any $a \in(0,1)$,

$$
S_{a}(\rho \| \sigma) \leq T(\rho, \sigma)
$$

Equality can be obtained for any value of $t=T(\rho, \sigma)$ in dimension 3 and higher by choosing $\rho=\operatorname{Diag}(t, 0,1-t)$ and $\sigma=\operatorname{Diag}(0, t, 1-t)$.

A second and unsuspected corollary is a strengthening of a very wellknown inequality (see, e.g. [8], Th. 3.7) for the entropy of an ensemble of two states: for any two states $\rho, \sigma$ and $(p, 1-p)$ a probability distribution,

$$
S(p \rho+(1-p) \sigma) \leq p S(\rho)+(1-p) S(\sigma)+h(p),
$$

where $h(p)=-p \log p-(1-p) \log (1-p)$ is the binary Shannon entropy. This inequality is equivalent to subadditivity of the von Neumann entropy (w.r.t. ordinary addition) for positive (non-normalised) operators: for any $A, B \geq 0$

$$
S(A+B) \leq S(A)+S(B) .
$$

Indeed, substituting $A=p \rho$ and $B=(1-p) \sigma$ yields (21).

The quantity $S(p \rho+(1-p) \sigma)-(p S(\rho)+(1-p) S(\sigma))$ is known as the Holevo quantity $\chi(\mathcal{E})$ for the ensemble $\mathcal{E}=\{(p, \rho),(1-p, \sigma)\}$ (of cardinality 2 ). The bound says that $\chi(\mathcal{E}) \leq h(p)$. Using Theorem 4 , we get a sharper bound: 
Corollary 2. For any ensemble $\mathcal{E}=\{(p, \rho),(1-p, \sigma)\}$ of cardinality 2,

$$
\chi(\mathcal{E}) \leq h(p) T(\rho, \sigma) .
$$

Proof. Let $\tau=p \rho+(1-p) \sigma$. Notice that $S(\tau)-(p S(\rho)+(1-p) S(\sigma))$ is equal to $p S(\rho \| \tau)+(1-p) S(\sigma \| \tau)$. Applying inequality (19) to both terms gives $-p \log (p) T(\rho, \sigma)-(1-p) \log (1-p) T(\rho, \sigma)$ as an upper bound.

Question. As inequality (21) immediately generalises to ensembles of any cardinality $([6]$, section 11.3.6), namely, $\chi(\mathcal{E}) \leq H(p)$ (where $H(p)$ is the Shannon entropy of the probability distribution of $\mathcal{E}$ ), it is fair to ask for a similar generalisation of the Corollary.

In [10], related upper bounds were studied. For cardinality 2, a bound was found in terms of the probability $p$ and the Uhlmann fidelity between $\rho$ and $\sigma, F=\|\sqrt{\rho} \sqrt{\sigma}\|_{1}$. For cardinality 3 , a generalisation was conjectured in [4]. For general cardinalities a bound was proven that is sharper than $H(p)$ and is expressed in terms of the so-called exchange entropy [10].

We now present the proof of Theorem 4. It relies on the properties of the Fréchet derivative of the matrix logarithm given in Section 2 ,

\section{Proof of Theorem 4 .}

Let $\rho$ and $\sigma$ be two given states, and $\tau=a \rho+(1-a) \sigma$. Define $s=(1-a) / a$, which is a non-negative number. Thus $\tau=a(\rho+s \sigma)$. W.l.o.g. we will assume that $\rho+s \sigma$ is full rank.

Let $\Delta:=\rho-\sigma, t:=T(\rho, \sigma)=\|\Delta\|_{1} / 2$ and $\omega:=\Delta / t$. Obviously, $\omega$ has trace 0 and trace norm 2 . Let its Jordan decomposition be $\omega=\omega_{+}-\omega_{-}$. Thus $\omega \leq \omega_{+}$and $\operatorname{Tr} \omega_{+}=\operatorname{Tr} \omega_{-}=1$.

Now consider the expression $s \operatorname{Tr} \omega \mathcal{T}_{\rho+s \sigma}(\sigma)$. Since $\mathcal{T}_{\rho+s \sigma}(\sigma) \geq 0$, and $\omega \leq \omega_{+}$, we have

$$
\begin{aligned}
s \operatorname{Tr} \omega \mathcal{T}_{\rho+s \sigma}(\sigma) & =\operatorname{Tr} \omega \mathcal{T}_{\rho+s \sigma}(s \sigma) \\
& \leq \operatorname{Tr} \omega_{+} \mathcal{T}_{\rho+s \sigma}(s \sigma) \\
& \leq \operatorname{Tr} \omega_{+} \mathcal{T}_{\rho+s \sigma}(\rho+s \sigma) \\
& =\operatorname{Tr} \omega_{+} \mathbb{1} \\
& =1 .
\end{aligned}
$$

Then, noting that $\rho=\sigma-t \omega$,

$$
\begin{aligned}
(1+s) \operatorname{Tr} \rho \mathcal{T}_{\rho+s \sigma}(\sigma) & =\operatorname{Tr}(\rho+s \rho) \mathcal{T}_{\rho+s \sigma}(\sigma) \\
& =\operatorname{Tr}(\rho+s \sigma-s t \omega) \mathcal{T}_{\rho+s \sigma}(\sigma)
\end{aligned}
$$




$$
\begin{aligned}
& =\operatorname{Tr}(\rho+s \sigma) \mathcal{T}_{\rho+s \sigma}(\sigma)-t s \operatorname{Tr} \omega \mathcal{T}_{\rho+s \sigma}(\sigma) \\
& =\operatorname{Tr} \sigma \mathcal{T}_{\rho+s \sigma}(\rho+s \sigma)-t s \operatorname{Tr} \omega \mathcal{T}_{\rho+s \sigma}(\sigma) \\
& =\operatorname{Tr} \sigma-t s \operatorname{Tr} \omega \mathcal{T}_{\rho+s \sigma}(\sigma) \\
& \geq 1-t
\end{aligned}
$$

Therefore,

$$
\operatorname{Tr} \rho \mathcal{T}_{\rho+s \sigma}(\sigma) \geq \frac{1-t}{1+s}
$$

Integrating over $s$ from 0 to $(1-a) / a$ then yields

$$
\operatorname{Tr} \rho \log (\rho+(1-a) \sigma / a)-\operatorname{Tr} \rho \log (\rho) \geq(1-t) \log (1 / a),
$$

which becomes, after adding $\log a$ to both sides,

$$
\operatorname{Tr} \rho \log (a \rho+(1-a) \sigma)-\operatorname{Tr} \rho \log (\rho) \geq t \log (a),
$$

which is equivalent to the statement of the Theorem.

\section{Cases of maximality}

The following theorem characterises those cases when the telescopic relative entropy achieves its maximal value of 1 .

Theorem 5. For any $a \in(0,1), S_{a}(\rho \| \sigma)=1$ iff $\rho \perp \sigma$.

Proof. We have $S_{a}(\rho \| \sigma)=1$ iff $\operatorname{Tr} \rho \log (a \rho)=\operatorname{Tr} \rho \log (a \rho+(1-a) \sigma)$ or, putting $X=a \rho$ and $Y=(1-a) \sigma$, iff $\operatorname{Tr} X \log X=\operatorname{Tr} X \log (X+Y)$. Since $X, Y \geq 0$, operator monotonicity of the logarithm gives $\operatorname{Tr} X \log (X+Y) \geq$ $\operatorname{Tr} X \log X$. We want to characterise the cases of equality. One direction is obvious; if $X$ and $Y$ are orthogonal, clearly we have equality.

To prove that there are no other possibilities, assume $\operatorname{Tr} X(\log (X+$ $Y)-\log X)=0$. Consider first the case $X>0$. Define $Z=\log (X+Y)-$ $\log X$. Because of monotonicity of the logarithm we have $Z \geq 0$, hence the assumption, $\operatorname{Tr} X Z=0$, implies $Z=0$, i.e. $\log (X+Y)=\log X$. As the logarithm is invertible on the set of positive operators, this can only be true iff $Y=0$.

Now consider the general case $X \geq 0$, and assume $X$ has a non-trivial kernel. Then we can decompose the Hilbert space $\mathcal{H}$ as the direct sum $\mathcal{H}=\operatorname{supp} X \oplus \operatorname{ker} X$. We have $X=\left.X\right|_{X} \oplus 0$, with $\left.X\right|_{X}>0$. W.l.o.g. we can assume that $X+Y>0$, so that its logarithm is well-defined. By the convention to take $\lim _{x \rightarrow 0} x \log x=0, \operatorname{Tr} X \log X$ is well-defined, too, and equal to $\left.\left.\operatorname{Tr} X\right|_{X} \log X\right|_{X}$. The assumption $\operatorname{Tr} X(\log (X+Y)-\log X)=0$ 
can then be written as $\left.\operatorname{Tr} X\right|_{X}\left(\left.\log (X+Y)\right|_{X}-\log \left(\left.X\right|_{X}\right)\right)=0$. Let us therefore define $Z=\left.\log (X+Y)\right|_{X}-\log \left(\left.X\right|_{X}\right)$.

As can be expected, $Z \geq 0$. To prove this, put $X^{\prime}=\left.X\right|_{X} \oplus \epsilon \mathbb{1}$. By operator monotonicity of the $\operatorname{logarithm}, \log \left(X^{\prime}+Y\right)-\log X^{\prime} \geq 0$, for all $\epsilon>0$. In particular, the compression to $\operatorname{supp} X$ is positive too: $\left.\log \left(X^{\prime}+Y\right)\right|_{X}-\left.\log \left(X^{\prime}\right)\right|_{X} \geq 0$. Since $X^{\prime}$ is defined as a direct sum of $X$ and $\epsilon \mathbb{1},\left.\log \left(X^{\prime}\right)\right|_{X}=\log \left(\left.X^{\prime}\right|_{X}\right)=\log \left(\left.X\right|_{X}\right)$. Since $\lim _{\epsilon \rightarrow 0} X^{\prime}+Y=X+Y$, we get, indeed, $\left.\log (X+Y)\right|_{X}-\log \left(\left.X\right|_{X}\right) \geq 0$.

The assumption reduces to $\left.\operatorname{Tr} X\right|_{X} Z=0$. Because $\left.X\right|_{X}>0$ and $Z \geq 0$, this implies $Z=0$.

This implies $\left.Y\right|_{X}=0$, so that, indeed, $Y$ must be orthogonal to $X$.

\section{Relative Renyi Entropies}

The relative Renyi entropies are parameterised modifications of the relative entropy given by

$$
\operatorname{Tr} \rho^{1-p} \sigma^{p},
$$

where $p$ is a real number. Here we restrict ourselves to the case $0 \leq p \leq 1$.

Just as we have done for the relative entropy, one can define the telescopic relative Renyi entropy, even though the problem of infinite values does not pose itself here; indeed, $\operatorname{Tr} \rho^{1-p} \sigma^{p}$ is always between 0 and 1. Nevertheless, some interesting relationships occur when telescoping the relative Renyi entropies. In particular, by exploiting the methods used in Section 4 we obtain a shorter and much simpler proof of an inequality already proven in $[1]$.

Let us therefore consider the quantity $\operatorname{Tr} \rho^{1-p}(a \rho+(1-a) \sigma)^{p}$. Firstly, let us determine its extremal values for fixed values of $a$. Clearly, the maximum is still 1 , achieved when $\rho=\sigma$. The minimal value, however, is now $a^{p}$. This follows easily from operator monotonicity of the fractional power $x \mapsto x^{p}$ when $0 \leq p \leq 1$. Indeed,

$$
\begin{aligned}
\operatorname{Tr} \rho^{1-p}(a \rho+(1-a) \sigma)^{p} & \geq \operatorname{Tr} \rho^{1-p}(a \rho)^{p} \\
& =a^{p} \operatorname{Tr} \rho^{1-p} \rho^{p}=a^{p} \operatorname{Tr} \rho=a^{p} .
\end{aligned}
$$

Equality can be achieved for orthogonal $\rho$ and $\sigma$.

Hence, we define the telescopic relative Renyi entropies (TRRE) as follows:

\section{Definition 2.}

$$
Q_{p, a}(\rho, \sigma)=\frac{1}{1-a^{p}}\left(1-\operatorname{Tr} \rho^{p}(a \rho+(1-a) \sigma)^{1-p}\right) .
$$


By the above, the TRRE has values between 0 and 1 .

We now show that a sharper upper bound is given by the trace norm distance between $\rho$ and $\sigma$.

\section{Theorem 6.}

$$
Q_{p, a}(\rho, \sigma) \leq T(\rho, \sigma)
$$

As a special case, we recover the bound $Q_{p, 0}(\rho, \sigma)=1-\operatorname{Tr} \rho^{p} \sigma^{1-p} \leq$ $T(\rho, \sigma)$, which was instrumental in proving optimality of the Chernoff bound in symmetric hypothesis testing [1].

Just as we did for the operator logarithm, we can define a linear map based on the Fréchet derivative of the fractional power function $x^{p}$, via

$$
\left.\frac{d}{d t}\right|_{t=0}(A+t \Delta)^{p}=: \mathcal{T}_{A ; p}(\Delta) .
$$

Since $x \mapsto x^{p}$ is a non-negative operator monotone function for $0 \leq p \leq 1$, the fractional power of a positive operator $A$ can be written as the integral

$$
A^{p}=\int_{0}^{\infty} d \mu_{p}(s)(A+s)^{-1} A
$$

where $d \mu_{p}(s)$ is a certain measure, parameterised by $p$, that is positive for $0 \leq p \leq 1$. Its Fréchet derivative is therefore given by

$$
\begin{aligned}
\left.\frac{d}{d t}\right|_{t=0}(A+t \Delta)^{p} & =\int_{0}^{\infty} d \mu_{p}(s)\left((A+s)^{-1} \Delta-(A+s)^{-1} \Delta(A+s)^{-1} A\right) \\
& =\int_{0}^{\infty} d \mu_{p}(s) s(A+s)^{-1} \Delta(A+s)^{-1} .
\end{aligned}
$$

Therefore, $\mathcal{T}_{A ; p}$ has the integral representation

$$
\mathcal{T}_{A ; p}(\Delta)=\int_{0}^{\infty} d \mu_{p}(s) s(A+s)^{-1} \Delta(A+s)^{-1} .
$$

From this representation we easily derive the following properties:

1. $\operatorname{Tr} X \mathcal{T}_{A ; p}(Y)=\operatorname{Tr} Y \mathcal{T}_{A ; p}(X)$ for any $X$ and $Y$;

2. the map $\mathcal{T}_{A ; p}$ preserves the positive definite ordering;

3. in particular, $\mathcal{T}_{A ; p}(B)$ is positive for positive $B$;

4. for $0<p<1, \mathcal{T}_{A ; p}\left(A^{1-p}\right)=p\{A\}$. 
The last property follows from

$$
\begin{aligned}
\mathcal{T}_{A ; p}\left(A^{1-p}\right) & =\left.\frac{d}{d t}\right|_{t=0}\left(A+t A^{1-p}\right)^{p} \\
& =p A^{p-1} A^{1-p}=p\{A\} .
\end{aligned}
$$

Using these properties, we can easily prove the theorem.

Proof of Theorem [6. Let $\Delta=\rho-\sigma$, and $t=T(\rho, \sigma)$ then $\Delta$ has Jordan decomposition $\Delta=t \omega_{+}-t \omega_{-}$, where $\omega_{+}$and $\omega_{-}$are orthogonal density operators. Then

$$
\begin{aligned}
\operatorname{Tr}(a \rho)^{1-p} \mathcal{T}_{a \rho+(1-a) \sigma ; p}(\Delta) & \leq \operatorname{Tr}(a \rho)^{1-p} \mathcal{T}_{a \rho+(1-a) \sigma ; p}\left(t \omega_{+}\right) \\
& \leq \operatorname{Tr}(a \rho+(1-a) \sigma)^{1-p} \mathcal{T}_{a \rho+(1-a) \sigma ; p}\left(t \omega_{+}\right) \\
& =\operatorname{Tr} t \omega_{+} \mathcal{T}_{a \rho+(1-a) \sigma ; p}\left((a \rho+(1-a) \sigma)^{1-p}\right) \\
& =\operatorname{Tr} t \omega_{+} p\{a \rho+(1-a) \sigma\} \\
& \leq p t
\end{aligned}
$$

In the first line we used the fact that $\Delta \leq t \omega_{+}$and property 2 ; in the second line we used operator monotonicity of $x^{1-p}$ and property 3 ; in the third line we used property 1 , and in the fourth property 4 . In the last line we used the fact that $\operatorname{Tr} X Y \leq 1$ when $X$ is a density operator and $Y$ is a projector.

Exploiting the inequality just obtained in the last of the following integrals, we get

$$
\begin{aligned}
1-\operatorname{Tr} \rho^{p}(a \rho+(1-a) \sigma)^{1-p} & =\operatorname{Tr} \rho^{1-p}\left(\rho^{p}-(a \rho+(1-a) \sigma)^{p}\right) \\
& =\int_{a}^{1} d a \frac{d}{d a} \operatorname{Tr} \rho^{1-p}(a \rho+(1-a) \sigma)^{p} \\
& =\int_{a}^{1} d a \operatorname{Tr} \rho^{1-p} \frac{d}{d a}(a \rho+(1-a) \sigma)^{p} \\
& =\int_{a}^{1} d a \operatorname{Tr} \rho^{1-p} \mathcal{T}_{a \rho+(1-a) \sigma ; p}(\rho-\sigma) \\
& \leq \int_{a}^{1} d a a^{p-1} p t \\
& =\left(1-a^{p}\right) t
\end{aligned}
$$

which is equivalent to the statement of the theorem. 


\section{Future work}

In forthcoming papers we will explore further properties of the telescopic relative entropy. One other problem with the ordinary relative entropy is the absence of a triangle inequality, in the sense that no useful upper bound exists on the difference $S\left(\rho \| \tau_{1}\right)-S\left(\rho \| \tau_{2}\right)$. Indeed, this difference can be infinite. It turns out that such a bound does exist for the telescopic relative entropy. Together with an upper bound on the difference $S\left(\rho_{1} \| \tau\right)-S\left(\rho_{2} \| \tau\right)$ it will be presented and proven in [2].

We will also study an interesting connection with Hamiltonian reconstruction. There is some evidence that the difference $S_{a}\left(\rho \| \tau_{1}\right)-S_{a}\left(\rho \| \tau_{2}\right)$

might provide non-trivial lower bounds on the time needed for state $\tau_{1}$ to evolve unitarily into state $\tau_{2}$ under the influence of a Hamiltonian with bounded energy.

\section{Acknowledgments}

The main part of this work was done at the Institut Mittag-Leffler, Djursholm (Sweden), during an extended stay at its Fall 2010 Semester on Quantum Information Theory.

\section{References}

1. K.M.R. Audenaert, M. Nussbaum, A. Szkoła and F. Verstraete, Commun. Math. Phys. 279, 251-283 (2008).

2. K.M.R. Audenaert, "Telescopic Relative Entropy - II: Triangle inequalities", arxiv:1102:3041 (2011).

3. N. Datta, "Min- and Max-Relative Entropies and a New Entanglement Monotone," IEEE Trans. Information Theory 55, 2816-2826 (2009).

4. M. Fannes, F. de Melo, W. Roga and K. Życzkowski, "Matrices of fidelities for ensembles of quantum states and the Holevo quantity", arXiv:1104.2271 (2011).

5. K. Lendi, F. Farhadmotamed and A.J. van Wonderen, "Regularization of quantum relative entropy in finite dimensions and application to entropy production", J. Stat. Phys. 92(5/6), 1115-1135 (1998).

6. M.A. Nielsen and I.L. Chuang, Quantum Computation and Quantum Information, Cambridge University Press (2000).

7. M. Ohya and D. Petz, "Quantum entropy and its use", Springer (1993).

8. D. Petz, Quantum Information Theory and Quantum Statistics, Springer-Verlag, Berlin (2008).

9. R. Renner, "Security of quantum key distribution," PhD thesis, ETH Zurich, arXiv:quant-ph/0512258 (2005).

10. W. Roga, M. Fannes and K. Życzkowski, Phys. Rev. Lett. 105, 040505 (2010). 